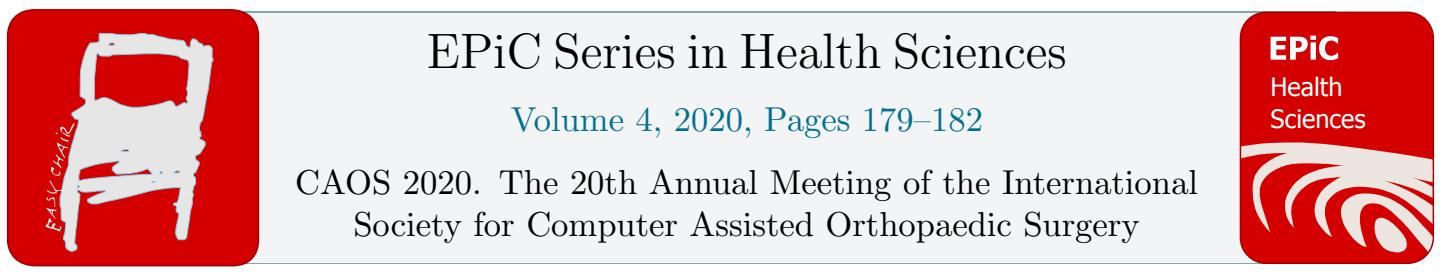

\title{
Functional Correctability of the Knee Joint Using 3D-to-2D Registration of X-Ray Imaging in Stressed Positions
}

\author{
Jialong $\mathrm{Li}^{1}$, David Liu ${ }^{2}$, Stephen McMahon ${ }^{3}$, Jonathan Bare ${ }^{3}$, Andrew \\ Shimmin ${ }^{3}$, Brad Miles ${ }^{4}$, Qing Li $^{1}$, Joshua Twiggs ${ }^{4}$ \\ ${ }^{1}$ School of Biomedical Engineering, Faculty of Aerospace, Mechanical and Mechatronics \\ Engineering, University of Sydney, NSW, Australia \\ ${ }^{2}$ The Gold Coast Centre for Bone and Joint Surgery, Palm Beach, Queensland, Australia \\ ${ }^{3}$ Melbourne Orthopaedic Group, Windsor, Victoria, Australia \\ ${ }^{4} 360$ Med Care, Pymble, NSW, Australia
}

\begin{abstract}
Correcting the knee joint to accommodate the pre-existing soft tissue structures is a major aim in total knee arthroplasty. 3D-to-2D registration of segmented boney geometry obtained from computer tomography (CT) scans to functionally stressed positions in X-Ray (XR) imaging can be a more accurate method of obtaining the laxity envelop. From which, a more patient specific limit for the correctability of the knee joint can be found. Supine CT scans were segmented, and 3D bone models and anatomical landmarks were registered to $2 \mathrm{D}$ functional stressed XR imaging. The functional position of the patient femoral and tibial bone is then used to calculate the varus and valgus extent of the patient's knee joint laxity. 103 preoperative knees undergoing primary total knee arthroplasty identified from six different surgeons. The range of patients who are correctable back to within 3 degrees when a force is applied is $52 \% .65 \%$ of patients who were within 3 degrees varus/valgus for tibia varus were correctable, while $41 \%$ of patients who were within 3 degrees varus/valgus for femoral valgus were correctable. $26 \%$ of patients were correctable when the LDFA is outside the threshold and MPTA is not, while $56 \%$ of patients were correctable when the MPTA is outside the threshold and LDFA is not. This study demonstrates the complexity of soft tissue structures of the knee joint. The main finding of this study is that correctability is more sensitive to the proximal tibial than the distal and posterior femoral articulating geometry. A lack of dependency between correctability of the knee joint and anatomical measures specific to flexion of the knee is seen. Careful consideration should be made intraoperatively when balancing the knee joint flexion gaps surrounding the soft tissue structures.
\end{abstract}


Functional Correctability of the Knee Joint using 3D-to-2D Registration of X-Ray Imaging ... Li et al.

\section{Introduction}

Correcting the knee joint to accommodate the pre-existing soft tissue structures is a major aim in total knee arthroplasty [1, 2]. Variation in knee joint anatomy and soft tissue mechanisms exist in population and can affect the intra-operative decision-making process and ultimately influence patient outcome. Regardless of the surgical delivery methods, a compromise is required between obtaining balanced knee joint kinematics and implant alignment. Alignment philosophies and surgical techniques can dictate the interaction between the implant and the surrounding soft tissue structures of the operative knee.

Previous algorithms for the correctability of knee joints have been based on arbitrary values [3], and shortcomings for the method of measurement have been highlighted [4, 5]. 3D-to-2D registration of segmented boney geometry obtained from computer tomography (CT) scans to functionally stressed positions in X-Ray (XR) imaging can be a more accurate method of obtaining the laxity envelop. From which, a more patient specific limit for the correctability of the knee joint can be found.

\section{Methods}

Supine pre-operative CT scans were captured for each patient. CT scan set of each patient is segmented using Simpleware Software (Exeter, United Kingdom) with anatomical landmarks identified on the generated 3D bone models. Functional x-ray imaging was obtained for each patient, with functional position using a TELOS KT-1000 (METAX, Germany) stress device. XR images were then taken when a varus and valgus stress is applied parallel to the sagittal plane of the tibia in knee joint extension at 20 degrees and flexion between 45 and 90 degrees. Segmented bones were then registered to each 2D obtained X-ray image using Mimics (Materialise, Belgium) R19 Research software. Landmarks acquired from the supine pre-operative CT scans to define patient specific anatomic axes were transformed to the $2 \mathrm{D}$ registered bone reference frame.

In terms of anatomical measures, laxity of the knee joint is defined by the change between the varus and valgus extents. Correctability window is defined between 3 degrees varus and 3 degrees valgus in HKA. The functional position of the patient femoral and tibial bone is then used to calculate the varus and valgus extent of the patient's knee joint laxity. Correlations between anatomical landmarks and laxity ranges were determined. Statistical analysis was conducted for variations in anatomical morphology measures such as lateral distal femoral angle (LDFA) and medial proximal tibial angle (MPTA) as well as their influences on correctability of a patient.

\section{Results}

103 preoperative knees undergoing primary total knee arthroplasty fitting the above selection criteria were identified from six different surgeons at three different sites. Median for the LDFA is $2.5 \mathrm{deg}$ valgus and MDFA are 3.3deg varus. The range of patients who are correctable back to within 3 degrees when a force is applied is $52 \% .65 \%$ of patients who were within 3 degrees varus/valgus for tibia varus were correctable, while $41 \%$ of patients who were within 3 degrees varus/valgus for 
femoral valgus were correctable. $26 \%$ of patients were correctable when the LDFA is outside the threshold and MPTA is not, while 56\% of patients were correctable when the MPTA is outside the threshold and LDFA is not, see figure 1.

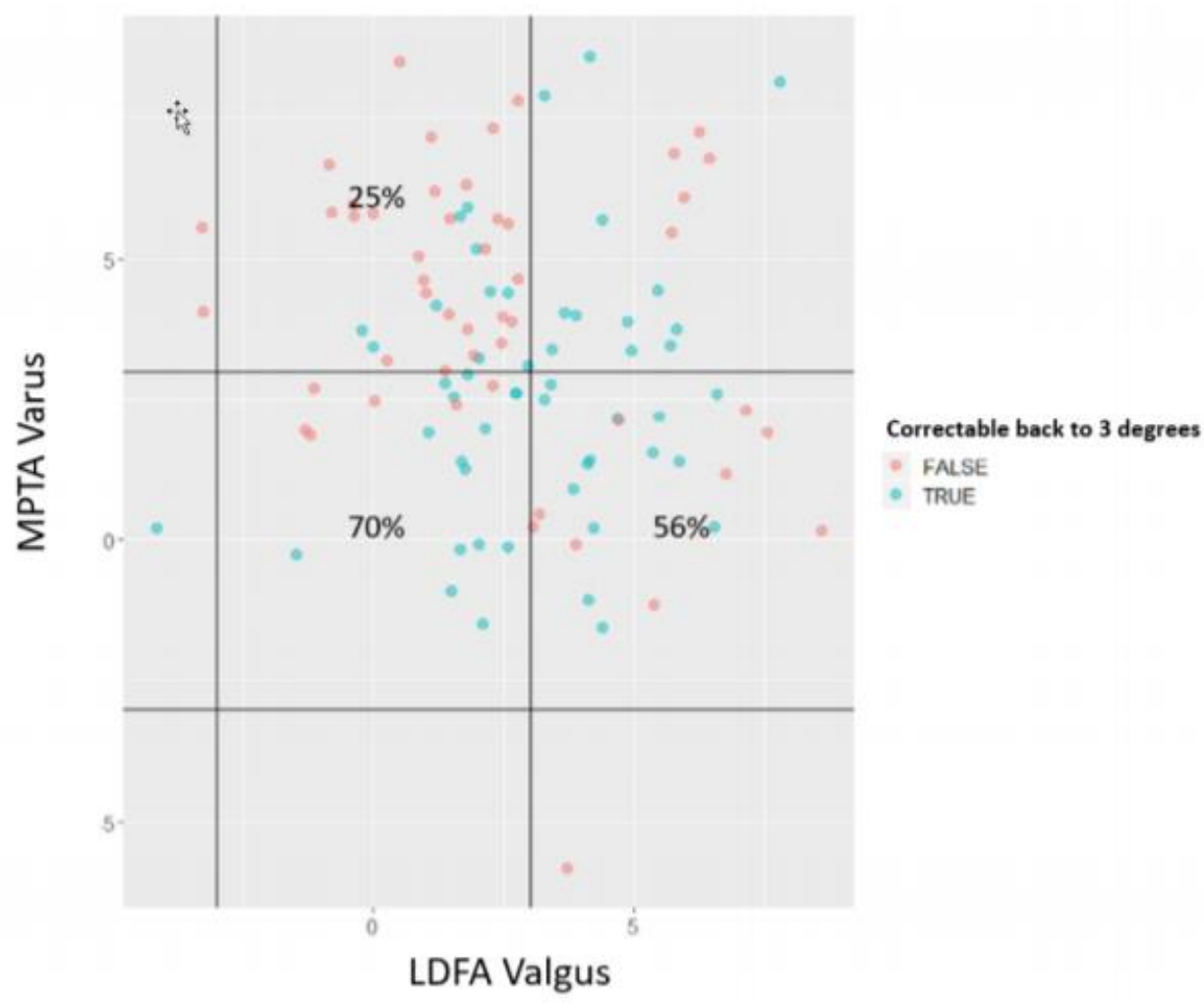

Figure 1. Correctability based on LDFA and MPTA anatomical morphology

No statistically significant difference in HKA alignment is observed between patients who are correctable and non-correctable. No statistically significant difference is seen between laxity range in knee flexion and PCA to TEA angle, LDFA and MPTA

\section{Discussion}

This study demonstrates the complexity of soft tissue structures of the knee joint. The main finding of this study is that correctability is more sensitive to the proximal tibial than the distal and posterior femoral articulating geometry. This study also highlighted the lack of dependency between correctability of the knee joint and anatomical measures specific to flexion of the knee. Controversy exist for the correct gap to target in the flexion space and can vary greatly based on surgical techniques and alignment philosophies [2, 6, 7].

Previous methods of defining correctability in patients can be binarizing and may not be appropriate for the wide variation of anatomy demonstrated in the present study [3]. Waldstein et al. 
Functional Correctability of the Knee Joint using 3D-to-2D Registration of X-Ray Imaging ... Li et al.

reported knees to be correctable to three degrees varus/valgus when the hip-to-ankle standing radiographs is 10 degrees or less to mechanical varus. Our results demonstrated a percentage of patients within the same group that were not correctable back to three degrees varus/valgus. The inclusion of natively valgus patients which were not included in the previous studies, as well as our use of 2D-3D registration of stressed functional positions, could be factor in the differences between ours and previously reported ligament laxity results.

While navigation is a reliable method of obtaining intraoperative knee joint laxity, the method is not fully representative of the patient's functional laxity envelop as the soft tissue structures are not innervated when patient is under anesthesia. Intraoperatively, determining the extent of correctability based on a patient's laxity envelop for the purposes of knee joint balancing is complex, as indicated by our results. Restoration of natural kinematics requires careful consideration between resection alignment and the patient specific ligament profiles.

\section{Conclusion}

This study shows correctability of the knee joint is more discretizing to the MPTA compared to LDFA. Careful consideration should be made intraoperatively when balancing the knee joint flexion gaps surrounding the soft tissue structures.

\section{References}

[1] H. Dinges, and H. Hommel, "IS CONSTITUTIONAL OR RESIDUELL VARUS ALREADY TODAY AN

ALTERNATIVE IN THE TKR." pp. 113-113.

[2] M. P. Abdel, S. Oussedik, S. Parratte, S. Lustig, and F. Haddad, "Coronal alignment in total knee replacement: historical review, contemporary analysis, and future direction," The bone \& joint journal, vol. 96, no. 7, pp. 857-862, 2014.

[3] W. Waldstein, J. B. Monsef, J. Buckup, and F. Boettner, "The value of valgus stress radiographs in the workup for medial unicompartmental arthritis," Clinical Orthopaedics and Related Research ${ }^{\circledR}$, vol. 471, no. 12, pp. 3998-4003, 2013.

[4] T. M. Kreitz, M. G. Maltenfort, and J. H. Lonner, "The valgus stress radiograph does not determine the full extent of correction of deformity prior to medial unicompartmental knee arthroplasty," The Journal of arthroplasty, vol. 30, no. 7, pp. 1233-1236, 2015.

[5] M. Norris, K. Gill, D. Karadaglis, and S. Chauhan, "THE ENVELOPE OF LAXITY AND BALANCING

OF TOTAL KNEE REPLACEMENTS USING NAVIGATION." pp. 426-426.

[6] N. C. Hunt, K. M. Ghosh, K. K. Athwal, L. M. Longstaff, A. A. Amis, and D. J. Deehan, "Lack of evidence to support present medial release methods in total knee arthroplasty," Knee

Surgery, Sports Traumatology, Arthroscopy, vol. 22, no. 12, pp. 3100-3112, 2014.

[7] J. Sikorski, "Alignment in total knee replacement," The Journal of bone and joint surgery.

British volume, vol. 90, no. 9, pp. 1121-1127, 2008. 